\title{
Vorwort zur 30. Auflage
}

Im Vordergrund der 1975 erschienenen Vorauflage, die sich einer lebhaften Nachfrage erfreute und recht bald nachgedruckt werden mußte, standen die Auswirkungen des 2. StrRG und des EGStGB. Beide Reformwerke haben erwartungsgemäß zu einer Fülle neuer Entscheidungen und wissenschaftlicher Veröffentlichungen geführt, deren Verarbeitung bereits für sich allein Anlaß zu einer Neuauflage gegeben hätte. In der Zwischenzeit ist die Strafrechtsreform nicht zum Stillstand gekommen. Mit dem 13., 14. und 15. Strafrechtsänderungsgesetz (Reform der Komplexe Unfallflucht, Terrorismusbekämpfung und Schwangerschaftsabbruch) sowie dem 1. Gesetz zur Bekämpfung der Wirtschaftskriminalität sind weitere Reformgesetze von weittragender Bedeutung in Kraft getreten, deren Einarbeitung den eigentlichen Schwerpunkt dieser Auflage darstellt. Die durch das 1. WiKG betroffenen Vorschriften (neue Tatbestände zur strafrechtlichen Erfassung des Subventions- und des Kreditbetrugs, Reform der Komplexe Konkursstrafrecht und Wucher) wurden von den Staatsanwälten Freund und Bieneck bearbeitet, die als Sachbearbeiter der bei der Staatsanwaltschaft Stuttgart eingerichteten Schwerpunktabteilung zur Bekämpfung der Wirtschaftskriminalität über eine mehrjährige Erfahrung auf diesem Gebiet verfügen.

Der Verfasser, der seit mehr als zwei Jahrzehnten in der Ausbildung des juristischen Nachwuchses tätig ist und den ehemaligen „Petters“ bereits seit der 1965 erschienenen 25. Auflage betreut, hat die Strafrechtsreformgesetze zum Anlaß genommen, alle von der Reform betroffenen Teile gründlich zu überarbeiten. In Fortführung der seit der 25. Auflage verfolgten Tendenz, das früher nur äußerst knapp gehaltene Erläuterungswerk in einen Kommentar umzugestalten, der allen Anforderungen in Studium und Praxis gerecht wird, wurden die Problemkreise, die erfahrungsgemäß für Studium und Praxis von besonderer Bedeutung sind, weiter ausgebaut und unter verstärkter Berücksichtigung von Rechtsprechung und Schrifttum wissenschaftlich vertieft. Das Manuskript wurde im Februar 1977 abgeschlossen. Spätere Veröffentlichungen konnten teilweise bis November 1977 eingearbeitet werden. Leider nicht mehr berücksichtigt werden konnte das Gesetz vom 22. 12. 1977 (BGBl. I 3104), wonach die Vorschriften über die Unterbringung in einer sozialtherapeutischen Anstalt nicht schon am 1. 1. 1978, sondern erst am 1. 1. 1985 in Kraft treten. Dem Leser wird empfohlen, dies in $\S 65$ Anm. I (S. 288) sowie auf den Seiten 282, 285, 289, 292, 293 und 294 handschriftlich zu vermerken.

Durch den Ausbau der Kommentierung erweiterte sich der Umfang des Werkes trotz erneuter Kürzung des Anhangs recht erheblich, was sich zwangsläufig auch auf der Kostenseite auswirken mußte. Die umfangreichen Änderungen gegenüber der Vorauflage ließen es geboten erscheinen, das Werk völlig neu zu setzen und bei dieser Gelegenheit auch in seiner äußeren 
Erscheinungsform zu modernisieren. Drucktechnische Hervorhebungen der wichtigsten Stichworte sollen das Werk noch übersichtlicher gestalten als bisher; der Sperrdruck wurde durch Kursivschrift ersetzt.

Abschließend möchte ich nicht versäumen, an dieser Stelle all denen zu danken, die mich durch ihre Anregungen bei der Vorbereitung des Manuskripts sowie durch ihre Hilfe bei der technischen Gestaltung der Neuauflage tatkräftig unterstützt haben. Mein Dank gilt insbesondere Herrn Rechtsreferendar Hermann Weber, der sich auch um die Korrekturen und das Sachregister verdient gemacht hat.

Heidelberg, im Dezember 1977

Holger Preisendanz

\section{Aus dem Vorwort zur 25. Auflage}

Der bisherige Herausgeber, Herr Landgerichtsrat Dr. Petters, verstarb am 18. 1. 1963, kurz nach Vollendung seines 75. Lebensjahres. Seinem Wunsch, sein Werk fortzuführen, bin ich gerne gefolgt. Dabei habe ich mich von dem Bestreben leiten lassen, Charakter und System des so beliebten Buchs zu erhalten. 